Case Report

\title{
Oral Melanotic Macule - An Unusual Occurrence In Gingiva
}

\author{
Sajna H.R. ${ }^{1}$, Biju Thomas ${ }^{2}$, Amitha Ramesh ${ }^{3}$, Rahul Bhandary ${ }^{4}$, Madhurya N. Kedlaya $^{5}$ \\ ${ }_{1,5}$ Post graduate student, ${ }^{2}$ Professor and HOD, ${ }^{3,4}$ Professor, Department of Periodontics, A. B. Shetty M emorial Institute of \\ Dental Sciences, Deralakatte, M angalore, NITTE (deemed to be University).
}

*Corresponding Author : Sajna H.R., Post graduate student, Department of Periodontics, A.B. Shetty Memorial Institute of Dental Sciences M angalore, NITTE (deemed to be University).

Received

: 04.10.2017

Review Completed : 12.12.2017

Accepted

: 05.01.2018

Keywords : pigmented lesions, melanin deposition, gingiva, oral melanotic macule.

\begin{tabular}{|c|}
\hline Access this article online \\
\hline Quick Response Code \\
\hline
\end{tabular}

\section{Introduction}

The term pigmentation refers to the deposition of coloring matter.The pigmented lesions found in the oral cavity represents a variety of clinical entities, ranging from physiologic changes to manifestations of systemic illnesses and malignant neoplasm.

There is very little information in literature about the brown or black melanotic lesions of the oral mucosas that are not a manifestation of racial pigmentation and are not associated with other syndromes. Normal pigmentation of the intraoral tissues is a relatively frequent finding, until recently very little attention has been paid to it $^{1}$.

Oral pigmentation may be exogenous or endogenous in origin. Exogenous pigmentation is commonly due to foreign-body implantation in the oral mucosa. Endogenous pigments include melanin, hemoglobin, hemosiderin and carotene. Melanin is the most commonly found endogenous pigment in the oral soft tissues ${ }^{1}$.
This is a case report of multifocal oral pigmentation in gingiva and labial mucosa which was diagnosed to be melanotic macule with clinico-histopathologic evidence. The Lesion is particularly interesting because of its occurrence in the gingiva.

\section{Case Report}

A 34 year old female patient reported to the Department of Periodontics with the chief complaint ofblack discoloration with respect to maxillary gingiva since one year.On eliciting the history, the patient reported that she had noticed the presence of black spot in the maxillary gingivawith respect to11 and 21 regions one year back which gradually increased in size. Patient was under medication for thyroid problem 6 months ago which she discontinued without the physicians consent. She didn't report of similar discolouration in any other region of the body.

On intraoral examination a bluish black discoloration of about $0.5 \times 0.3 \mathrm{~cm}$ which extends from the marginal gingiva 
towards the attached gingiva between 11 and 21 regions was noted. Similar discoloration was found in the labial mucosa of lower lip. The lesion was flat, soft, smooth and not tender with irregular border. There wasno signs of any discharge or elevation and radiographic examination did not show any evidence of hard tissue lesion. Therefore a provisional diagnosis of Oral melanotic maculae was made.

Thorough scaling and root planning along with palliative treatment was given and an excisional biopsy was performed using scalpel and blade under aseptic conditions. The tissue was stored in formalin solution and the specimen was sent for histo pathological examination to the Department of Oral Pathology. Post - operative instructions were given and the patient was recalled after two weeks for check up. After two weeks, uneventful healing was observed.

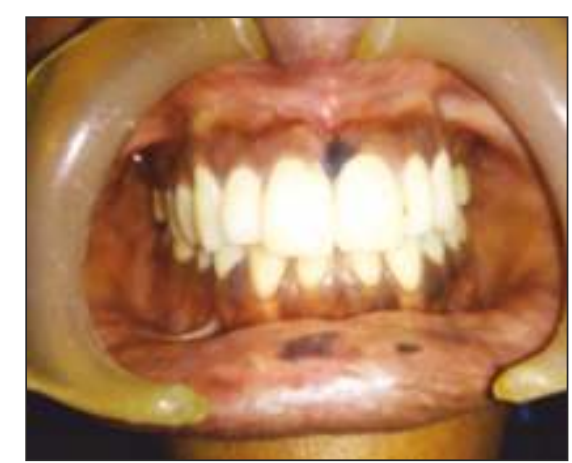

Figure 1

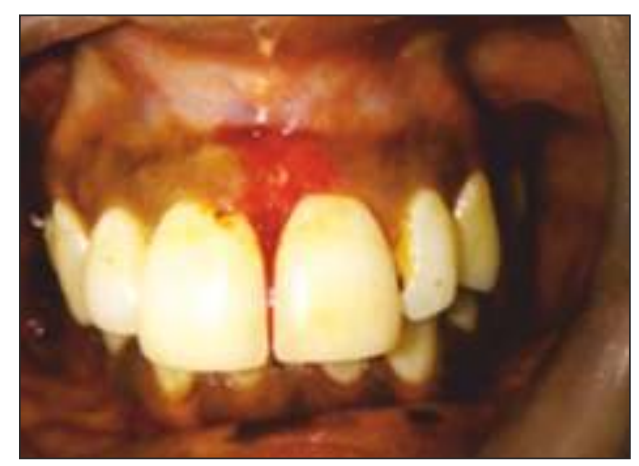

Figure 2

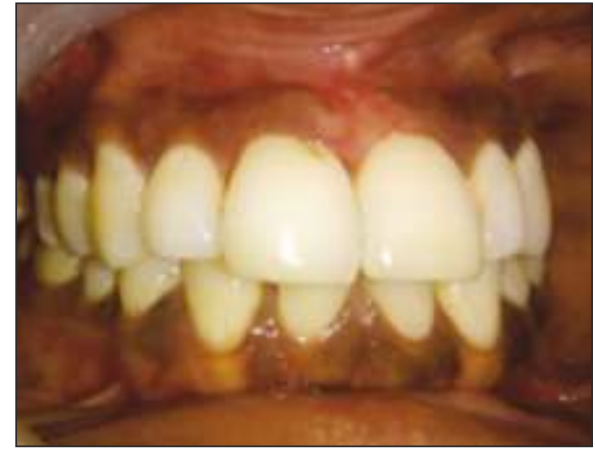

Figure 3

$H$ and $E$ stained section shows stratified squamous parakeratinised epithelium with long rete ridges and diffuse distribution of melanin pigmentation is seen all over the basal and parabasal layer of the epithelium. Underlying connective tissue contains loosely arranged collagen fibres, blood vessels and focal collection of inflammatory cells. Histo pathological examination revealed the lesion as M elanotic maculae.

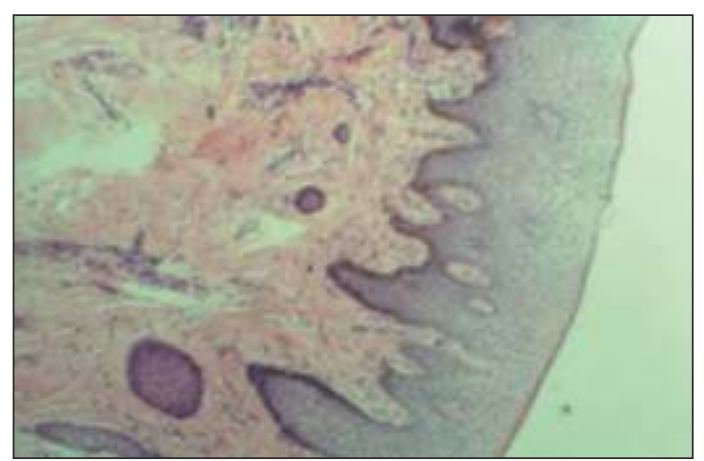

Figure 4

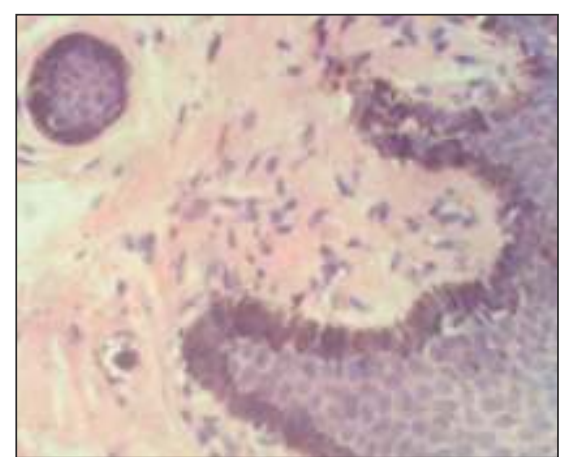

Figure 5

\section{Discussion}

Multifocal / diffuse pigmentation can occur in any individual. This type of pigmentation occurring in a healthy individual with dark complexion can be considered as a normal physiological pigmentation. But if the individual is 
systemically unhealthy occurrence of such pigmentation should be considered pathological. Various factors associated with the pigmentations in oral cavity includes Peutz-jeghers syndrome, McCune-Albright syndrome, environmental factors such as smokers melanosis, chronic cheek bite and systemic medications such as estrogen, chloroquine ${ }^{3}$.

The oral melanotic macule is a flat brown mucosal discoloration produced by focal increase in melanin deposition or a concomitant increase in the number of melanocytes. Females are most commonly affected than males with a ratio of 2:1. Usually located on the vermilion border of the lips also called as labial melanotic maculae (33\% of cases) followed by buccal mucosa, gingival and palate. ${ }^{2}$ Typical lesion appears as solitary well demarcated uniformly tan to dark brown asymptomatic round oval macule with a diameter of $7 \mathrm{~mm}$ or less. Lesions may be blue or black occasionally. The melanotic maculae have been variously termed as ephelis, melanosis, lentigo, solitary labial lentigo ${ }^{6}$, labial melanotic maculae and oral melanotic maculae. Ephelis (freckle) is a circumscribed brown macule present over skin that has been exposed to sunlight. Buchner and Hansen ${ }^{4}$ analyzed 105 cases of oral melanotic maculae and found that in most of the patients, melanotic macule was a solitary lesion and the most likely location was the vermilion border, followed by the gingival. The eitiology of melanotic maculae suggests that it may result from racial pigmentation, endocrine disturbance, anti-malarial therapy, Peutz-Jeghers syndrome, trauma, hemo-chromatosis, or chronic pulmonary disease, or they may be idiopathic. Majority of these require clinic pathologic correlation for definitive diagnosis. In these instances, the diagnosis should reflect the etiology. ${ }^{3}$

Histo-pathologically, the dark colour of the lesion is due to increase in melanin pigment of the basal cell layer, not from an increased number of melanocytes. M elanin may also be found in the lamina propria. Further histologic criteria are absence of elongated rete ridges and lack of prominent melanocytic activity. If there is an elogation of rete ridges, a heavily pigmented basal cell layer, and an increase in the number of normal-appearing basal layer melanocytes, ajunctional nevus has to be considered. If the melanocytes show proliferation, atypia, and some irregularity in their arrangement, the histopathologic diagnosis is atypical melanocytic hyperplasia, which may correspond clinically to early malignant melanoma. ${ }^{\text {? }}$

The small size, slow growth rate, and flat clinical appearance favour a benign diagnosis. ${ }^{3}$

Oral melanotic macule has to be differentiated from certain other similar conditions exhibiting hyperpigmentation such as racial pigmentation, post inflammatory hyper pigmentation, endocrinal hyper pigmentations, Anti-malarial drugs, Syndromes such as Peutz-Jeghers syndrome where freckles are seen not only in the oral cavity, but also at the distal extremities, Leopard syndrome where pigmentation is seen all over the body. ${ }^{3}$

In the present case report even though the patient is giving a history of intake of thyroid medication, she failed to produce the physician's reports or the medications which she had taken for few months.On blood investigation her present T3,T4,TSH values are within normal limits.The lesion present in lower labial mucosa much more resembles like oral melanotic maculae. The diagnosis of the pigmentation present in the gingiva was questionable but histo-pathological examination revealed the diffuse distribution of melanin pigmentation all over the basal and parabasal layer of the epithelium suggestive of oral melanotic macule.

The treatment of the oral melanotic macule is debatable ${ }^{5}$. The lesion is generally considered as a benign lesion with no malignant potential. But an early melanoma can have a similar clinical appearance. It is difficult to distinguish it clinically from other pigmented lesions, such as nevus, malignant melanoma in situ, and incipient malignant melanoma.

Complete excision of oral melanotic macule is indicated and frequent clinical examination for any change in the size, shape, or colouris recommended. This is especially necessary for lesions of the palate - a location for which oral 
malignant melanoma has a strong predilection. M elanotic lesions having a duration of fewer than 5 years, which have exhibited changes in size or colour or which exhibit tumefaction, ulceration, or bleeding, should be excised. The lesions with reliable history of more than 5 years

\section{References}

1. Dummett CO, Barens G. Pigmentation of the oral tissues: a review of the literature. Journal of periodontology. 1967 Sep;38(5):369-78.

2. Neville BW, Damm DD, Chi AC, Allen CM. Oral and maxillofacial pathology. Elsevier Health Sciences; 2015.

3. Pais S, Hegde SK, Bhat S. Oral melanotic macule-a case report. J Indian SocPedodPrev Dent. 2004 Jun 1;22(2):73-5.

4. Buchner A, Hansen LS. Melanotic macule of the oral mucosa: a clinicopathologic study of 105 cases. Oral Surgery, Oral M edicine, Oral Pathology. 1979 Sep 1;48(3):244-9.

5. Trodohl J. N. and Spragul W. G. : Benign and Malignant Mucosa, an Analysis of 135 cases. Cancer $1970 ; 25: 812$ - 823 without change in character in which a known cause seems

evident (trauma, etc.) is kept under observation. ${ }^{3}$ Hence in the present case, the patient is kept under regular follow up.

6. Beeker S. W. Jr. :Pigmentary Lesions oral melanotic macule on to the skin and oral cavity. Oral surg1969; 28:526-533.

7. Bork. K., Hoede. N., Korting. G. W., Burgdorf. W. H. C. and Young S. K. : Diseases of the oral M ucosa and the Lips. $2^{\text {nd }}$ Edition, W. B. Saunders Company, 1996; 310-312

8. Chaudhary A. P., Hampel A. and Gorlin R. L. : Primary Malignant Melanoma of the Oral Cavity-a Review of 105 cases. Cancer 1958; 11 : 923- 928

9. .Bengel W., Veltman G., Loevy H. T. and Pierangelo T. : Differential Diagnosis of Diseases of the Oral Mucosa. Quintessence Publishing Co., Inc., Chicago / Illin1989:153- 201 